\title{
DIRECTION OF VORTICITY AND A NEW REGULARITY CRITERION FOR THE NAVIER-STOKES EQUATIONS
}

\author{
YONG ZHOU ${ }^{1}$
}

(Received 25 September, 2003; revised 12 January, 2004)

\begin{abstract}
In this paper, we prove a new regularity criterion in terms of the direction of vorticity for the weak solution to 3-D incompressible Navier-Stokes equations. Under the framework of Constantin and Fefferman, a more relaxed regularity criterion in terms of the direction of vorticity is established.
\end{abstract}

\section{Introduction}

We consider the following Cauchy problem for the incompressible Navier-Stokes equations in $\mathbb{R}^{3} \times[0, T]$ :

$$
\left\{\begin{array}{l}
\frac{\partial u}{\partial t}+u \cdot \nabla u+\nabla p=\Delta u, \\
\operatorname{div} u=0, \\
u(x, 0)=u_{0}(x),
\end{array}\right.
$$

where $u=\left(u_{1}(x, t), u_{2}(x, t), u_{3}(x, t)\right)$ is the velocity field, $p(x, t)$ is a scalar pressure, and $u_{0}(x)$ with $\operatorname{div} u_{0}=0$ in the sense of distribution is the initial velocity field.

The study of the incompressible Navier-Stokes equations in three space dimensions has a long history. In the pioneering works [4] and [3], Leray and Hopf proved the existence of weak solutions $u(x, t) \in L^{\infty}\left(0, T ; L^{2}\left(\mathbb{R}^{3}\right)\right) \cap L^{2}\left(0, T ; H^{1}\left(\mathbb{R}^{3}\right)\right)$ for any given $u_{0}(x) \in L^{2}\left(\mathbb{R}^{3}\right)$. But the uniqueness and regularity of the Leray-Hopf weak solutions are still big open problems. In [2], they considered the direction of vorticity

$$
\xi(x, t)=\frac{\omega(x, t)}{|\omega(x, t)|}
$$

\footnotetext{
'Department of Mathematics, East China Normal University, Shanghai 200062, China; e-mail: yzhou@math.ecnu.edu.cn.

(C) Australian Mathematical Society 2005, Serial-fee code 1446-1811/05
} 
and proved that the solution of the Navier-Stokes equations (1.1) corresponding to $u_{0}$, which is divergence-free, smooth and has compact support, is strong and hence smooth $\left(C^{\infty}\right)$ on the time interval $[0, T)$ if the following assumption holds.

Assumption (A). There exist constants $K>0$ and $\rho(t)>0$ such that

$$
\left|P_{\xi(x, t)}^{\perp}(\xi(x+y, t))\right| \leq \frac{|y|}{\rho(t)} \quad \text { for } \rho(t)^{-12} \in L^{1}(0, T)
$$

holds if both $|\omega(x, t)|>K$ and $|\omega(x+y, t)|>K$, and $0 \leq t \leq T$, where $P_{\xi(x, t)}^{\perp}(\xi(x+y, t))$ denotes the projection of $\xi(x+y, t)$ orthogonal to $\xi(x, t)$.

In this paper, we want to prove regularity under a more relaxed assumption than (1.3). Our assumption reads as follows.

(H) There exist $\beta \in[1 / 2,3 / 2)$, a positive constant $K$, and $g(x, t) \in L^{\alpha, \gamma} \equiv$ $L^{\alpha}\left(0, T ; L^{\gamma}\left(\mathbb{R}^{3}\right)\right)$ such that

$$
\left|P_{\xi(x, t)}^{\perp}(\xi(x+y, t))\right| \leq g(x, t)|y|^{\beta}
$$

holds in the region both $|\omega(x, t)|>K$ and $|\omega(x+y, t)|>K$, and $0 \leq t \leq T$, with

$$
\frac{2}{\alpha}+\frac{3}{\gamma} \leq \beta-\frac{1}{2} \text { for } \alpha \in\left[\frac{4}{2 \beta-1}, \infty\right] \text {. }
$$

The main result of this paper is given in the following theorem.

THEOREM 1.1. Let $u_{0} \in H^{1}\left(\mathbb{R}^{3}\right)$ with $\operatorname{div} u_{0}=0$. Suppose $u$ is a Leray-Hopf weak solution to (1.1) corresponding to $u_{0}$. If $(\mathrm{H})$ is satisfied, then $u$ is a strong solution on $[0, T]$.

REMARK 1.1. We explain the motivation for establishing (1.4) as follows. First, from a mathematical viewpoint, $P_{\xi(x, t)}^{\perp}(\xi(x+y, t))$ is a function which depends on $x, y$ and $t$, so it is reasonable to find a condition in terms of $x, y$ and $t$ also to control it; that is, $g(x, t)$ should depend on both $x$ and $t$. In this sense, (1.4) is a more natural (and relaxed) condition than (1.3). Secondly, it is well known that if $u$ solves the Navier-Stokes equations, then so does $u_{\lambda}$ for all $\lambda>0$, where $u_{\lambda}(x, t)=\lambda u\left(\lambda x, \lambda^{2} t\right)$. So the ratio of the dimension of space to time is $3 / 2$ [1]. So the condition on $g(x, t)$ is $g \in L^{\alpha . \gamma}$ with $2 / \alpha+3 / \gamma$. Finally, there is a balance between $g(x, t)$ and $\beta$. When $\beta$ is bigger, the condition on $g(x, t)$ is more relaxed. Hence we let $\beta \in[1 / 2,3 / 2)$.

REMARK 1.2. One can find that even for $\beta=1$, assumption $(H)$ is weaker than assumption (A). When $\beta=1 / 2,(1.5)$ implies that $g(x, t) \in L^{\infty}\left(\mathbb{R}^{3} \times(0, T)\right)$.

REMARK 1.3. For recent progress on regularity criteria in terms of velocity and pressure, see [6-8] and references therein. 
Before going to the proof, let us recall the definition of Leray-Hopf weak solutions.

DEFinition. A measurable vector $u$ is called a Leray-Hopf weak solution to the Navier-Stokes equations (1.1), if $u$ satisfies the following properties:

(i) $u$ is weakly continuous from $[0, \infty)$ to $L^{2}\left(\mathbb{R}^{3}\right)$.

(ii) $u$ verifies (1.1) in the sense of distribution, that is,

$$
\int_{0}^{\infty} \int_{\mathbb{R}^{3}}\left(\frac{\partial \phi}{\partial t}+(u: \nabla) \phi\right) u d x d t+\int_{\mathbb{R}^{3}} u_{0} \phi(x, 0) d x=\int_{0}^{\infty} \int_{\mathbb{R}^{3}} \nabla u: \nabla \phi d x d t
$$

for all $\phi \in C_{0}^{\infty}\left(\mathbb{R}^{3} \times[0, \infty)\right)$ with $\operatorname{div} \phi=0$. Here $A: B=\sum_{t, j}^{3} a_{l j} b_{i j}, A=\left(a_{i j}\right)$ and $B=\left(b_{i j}\right)$ are $3 \times 3$ matrices and

$$
\int_{0}^{\infty} \int_{\mathbb{R}^{3}} u \cdot \nabla \phi d x d t=0
$$

for every $\phi \in C_{0}^{\infty}\left(\mathbb{R}^{3} \times[0, \infty)\right)$.

(iii) The energy inequality holds, that is,

$$
\|u(\cdot, t)\|_{L^{2}}^{2}+2 \int_{0}^{t}\|\nabla u(\cdot, s)\|_{L^{2}}^{2} d s \leq\left\|u_{0}\right\|_{L^{2}}^{2}, \quad t \geq 0 .
$$

By a strong solution we mean a weak solution $u$ such that

$$
u \in L^{\infty}\left(0, T ; H^{1}\right) \cap L^{2}\left(0, T ; H^{2}\right) .
$$

It is well known that strong solutions are regular (say, classical) and unique in the class of weak solutions.

\section{Proof of Theorem 1.1}

Since the theorem is proved under the framework of [2], let us recall a few observations regarding the relationship between divergence-free velocities, the associated vorticities and strain matrices in [2].

Let $\omega$ be the vorticity, $w=\operatorname{curl} u$. The strain matrix $S(x)$ in terms of $\omega$ is given by

$$
S(x)=S[\omega](x) \equiv \frac{1}{2}\left(\nabla u+(\nabla u)^{T}\right)=\frac{3}{4 \pi} \mathrm{P} . \mathrm{V} \cdot \int_{\mathbb{R}^{3}} M(\hat{y}, \omega(x+y)) \frac{d y}{|y|^{3}},
$$

where $M(\hat{y}, \omega)=[\hat{y} \otimes(\hat{y} \times \omega)+(\hat{y} \times \omega) \otimes \hat{y}] / 2$ and $\hat{y}=y /|y|$. Let

$$
\eta(x)=S(x) \xi(x) \cdot \xi(x)
$$


where $\xi$ is the direction of the vorticity defined by (1.2). Both $\eta$ and $\xi$ are defined in the region $\{x:|\omega(x)|>0\}$. It was derived in [2] that

$$
\eta(x)=\frac{3}{4 \pi} \text { P. V. } \int_{\mathbb{R}^{3}} D(\hat{y}, \xi(x+y), \xi(x))|\omega(x+y)| \frac{d y}{|y|^{3}},
$$

where $D$ is given by $D\left(e_{1}, e_{2}, e_{3}\right)=\left(e_{1} \cdot e_{3}\right)\left(\operatorname{Det}\left(e_{1}, e_{2}, e_{3}\right)\right)$. The Det in $D$ is the determinant of the matrix whose columns are the three unit column vectors $e_{1}, e_{2}$ and $e_{3}$. The geometric significance of $D$ is that it is a multiple of the volume of the prism of edges equal to $\hat{y}, \xi(x+y), \xi(x)$. In particular, it depends on $\xi(x+y)$ only through $P_{\xi(x)}^{\perp} \xi(x+y)$, thus

$$
|D(\hat{y}, \xi(x+y), \xi(x))| \leq\left|P_{\xi(x)}^{\perp} \xi(x+y)\right| .
$$

For solutions of the Navier-Stokes equations the dynamical significance of the expression

$$
(S(x, t) \omega(x, t)) \cdot \omega(x, t)=\eta(x, t)|\omega(x, t)|^{2}
$$

is that it presents the stretching term in evolution of the vorticity magnitude:

$$
\left(\partial_{t}+u \cdot \nabla-\Delta\right)|\omega|^{2}+|\nabla \omega|^{2}=\eta|\omega|^{2} .
$$

Equation (2.2) allows one to understand how local alignment of the vorticity direction depletes the nonlinearity.

After this review of important formulas we turn our attention to the proof. As in the argument in [7], one must have an a priori estimate for the strong solution under the assumption $(\mathrm{H})$. The key lemma reads as follows.

LEMMA 2.1. Let $u_{0} \in H^{1}\left(\mathbb{R}^{3}\right)$ with $\operatorname{div} u_{0}=0$. Suppose that $u$ is a strong solution on $(0, T)$. If $(\mathrm{H})$ is satisfied, then for all $0 \leq t \leq T$,

$$
\begin{aligned}
\|\omega\|_{L^{2}}^{2}+\int_{0}^{l}\|\nabla \omega\|_{L^{2}}^{2} d \leq & \left\|\omega_{0}\right\|_{L^{2}}^{2} \exp \left\{A T+B T^{3 / 5}+C\|g\|_{L^{\alpha, \gamma}}^{2 \alpha /(\alpha+2)}\right\} \\
& \times\left(1+A T+B T^{3 / 5}+C\|g\|_{L^{\alpha, \gamma}}^{c 2 \alpha /(\alpha+2)}\right),
\end{aligned}
$$

where the constant $A$ depends on $K, B$ depends on $K$ and $\left\|u_{0}\right\|_{L^{2}}$, while $C$ depends on $\alpha, \gamma$ and $\left\|u_{0}\right\|_{L^{2}}$.

ProOF. The vorticity field satisfies

$$
\left\{\begin{array}{l}
\frac{\partial \omega}{\partial t}+(u \cdot \nabla) \omega=(\omega \cdot \nabla) u+\Delta \omega \\
\operatorname{div} u=0 \\
\operatorname{curl} u=\omega \\
\omega(x, 0)=\omega_{0}(x)
\end{array}\right.
$$


Multiplying the first equation of (2.4) by $\omega$, and integrating on $\mathbb{R}^{3}$, after suitable integration by parts, we obtain

$$
\frac{1}{2} \frac{d}{d t}\|\omega(\cdot, t)\|_{L^{2}}^{2}+\|\nabla \omega(\cdot, t)\|_{L^{2}}^{2}=\int_{\mathbb{R}^{3}}(S(x, t) \omega(x, t)) \cdot \omega(x, t) d x \equiv I .
$$

Let $K$ be the positive constant in $(\mathrm{H})$ and split $\omega(x, t)$ as

$$
\begin{aligned}
\omega(x, t) & =\chi\left(\frac{|\omega(x, t)|}{K}\right) \omega(x, t)+\left(1-\chi\left(\frac{|\omega(x, t)|}{K}\right)\right) \omega(x, t) \\
& =w_{1}(x, t)+w_{2}(x, t),
\end{aligned}
$$

where the smooth bump function $\chi(\lambda) \in[0,1]$ is identically equal to one for $0 \leq \lambda \leq 1$ and identically equal to zero for $\lambda \geq 2$ or $\lambda \leq-1$.

So we can decompose $I$ into

$$
\begin{aligned}
I= & \int_{\mathbb{R}^{3}}(S(x, t) \omega(x, t)) \cdot \omega(x, t) d x \\
= & \sum_{i=1}^{2} \int_{\mathbb{R}^{3}} \sum_{k=1}^{2}\left(S_{i}(x, t) \omega_{1}(x, t)\right) \cdot \omega_{k}(x, t) d x \\
& +\sum_{i=1}^{2} \int_{\mathbb{R}^{3}}\left(S_{i}(x, t) \omega_{2}(x, t)\right) \cdot \omega_{1}(x, t) d x \\
& +\int_{\mathbb{R}^{3}}\left(S_{1}(x, t) \omega_{2}(x, t)\right) \cdot \omega_{2}(x, t) d x \\
& +\int_{\mathbb{R}^{3}}\left(S_{2}(x, t) \omega_{2}(x, t)\right) \cdot \omega_{2}(x, t) d x \equiv I_{1}+I_{2}+I_{3}+I_{4},
\end{aligned}
$$

where $S_{i}(x)=S\left[w_{i}\right](x)$, for $i=1,2$. We will estimate the above terms one by one:

$$
\begin{aligned}
\left|I_{1}\right| & =\left|\sum_{i=1}^{2} \int_{\mathbb{R}^{3}} \sum_{k=1}^{2}\left(S_{i}(x, t) \omega_{1}(x, t)\right) \cdot \omega_{k}(x, t) d x\right| \\
& \leq 2 K \sum_{i=1}^{2} \sum_{k=1}^{2}\left\|S_{i}(x, t)\right\|_{L^{2}}\left\|\omega_{k}\right\|_{L^{2}} \leq C_{1}\|\omega\|_{L^{2}}^{2}
\end{aligned}
$$

where $C_{1}$ is a constant depending only on $K$, and we used Hölder's inequality $\left|\omega_{1}\right| \leq 2 K$ and the Calderón-Zygmund inequality

$$
\left\|S_{i}(x, t)\right\|_{L^{q}} \leq C_{2}\left\|w_{\imath}\right\|_{L^{q}}
$$

with $1<q<\infty$ and $i=1,2$, with $C_{2}$ a constant depending only on $q$. 
The term $I_{2}$ can be treated similarly as $I_{1}$, so that

$$
\left|I_{2}\right| \leq C_{1}\|\omega\|_{L^{2}}^{2} .
$$

Also $I_{3}$ is not a difficult term, and it can be treated as

$$
\begin{aligned}
\left|I_{3}\right| & =\left|\int_{\mathbb{R}^{3}}\left(S_{1}(x, t) \omega_{2}(x, t)\right) \cdot \omega_{2}(x, t) d x\right| \\
& \leq C_{2}\left\|\omega_{1}\right\|_{L^{4}}\|\omega\|_{L^{4}}\|\omega\|_{L^{2}} \quad \text { (Hölder's inequality and (2.7)) } \\
& \leq C_{3}\left\|\omega_{1}\right\|_{L^{4}}\|\omega\|_{L^{2}}^{1 / 4}\|\nabla \omega\|_{L^{2}}^{3 / 4}\|\omega\|_{L^{2}} \quad \text { (the Gagliardo-Nirenberg inequality) } \\
& \leq \frac{1}{4}\|\nabla \omega\|_{L^{2}}^{2}+C_{4}\left\|\omega_{1}\right\|_{L^{4}}^{8 / 5}\|\omega\|_{L^{2}}^{2} \quad \text { (Young's inequality) } \\
& \leq \frac{1}{4}\|\nabla \omega\|_{L^{2}}^{2}+C_{5}\|\omega\|_{L^{2}}^{4 / 5}\|\omega\|_{L^{2}}^{2} .
\end{aligned}
$$

In the last inequality, we used the $L^{\infty}$-bound of $\left|\omega_{1}\right|$. We note that $C_{5}$ depends only on $K$.

Actually, as one can see, $I_{4}$ is the crucial term. First, note that

$$
\xi_{2}(x, t)=\frac{\omega_{2}(x, t)}{\left|\omega_{2}(x, t)\right|}=\xi(x, t),
$$

just as was done in $[2, \mathrm{pp} .785]$. Then

$$
\begin{aligned}
& \left|\left(S_{2}(x, t) \omega_{2}(x, t)\right) \cdot \omega_{2}(x, t)\right| \\
& \quad=\left|\omega_{2}(x, t)\right|^{2}\left|S_{2}(x, t) \xi_{2}(x, t) \cdot \xi_{2}(x, t)\right| \\
& \quad=\frac{3}{4 \pi}\left|\omega_{2}(x, t)\right|^{2}\left|\mathrm{P} . \mathrm{V} \cdot \int_{\mathbb{R}^{3}} D\left(\hat{y}, \xi_{2}(x+y, t), \xi_{2}(x, t)\right)\right| \omega_{2}(x+y, t)\left|\frac{d y}{|y|^{3}}\right| \\
& \quad \leq \frac{3}{4 \pi}|\omega(x, t)|^{2}|g(x, t)| f(x, t), \quad(\text { by }(1.4) \text { and }(2.1)),
\end{aligned}
$$

where $f(x, t)=\int_{\mathbf{R}^{3}}\left(|\omega(x+y, t)| /|y|^{3-\beta}\right) d y$. Therefore, due to (2.10), $I_{4}$ can be estimated as

$$
\begin{aligned}
\left|I_{4}\right| \leq & \frac{3}{4 \pi} \int_{\mathbb{R}^{3}}|\omega(x, t)|^{2}|g(x ; t)| f(x, t) d x \\
\leq & \frac{3}{4 \pi}\|\omega\|_{L^{a}}^{2}\|f\|_{L^{b}}\|g\|_{L^{r}} \quad \text { (Hölder's inequality) } \\
\leq & C_{6}\|\omega\|_{L^{a}}^{2}\|\omega\|_{L^{r}}\|g\|_{L^{r}} \quad \text { (the Hardy-Littlewood-Sobolev inequality) } \\
\leq & C_{7}\|\omega\|_{L^{2}}^{2(1-\theta)}\|\nabla \omega\|_{L^{2}}^{2 \theta}\|\omega\|_{L^{2}}^{1-\delta}\|\nabla \omega\|_{L^{2}}^{\delta}\|g\|_{L^{r}} \\
& \text { (the Gagliardo-Nirenberg inequality for }\|\omega\|_{L^{a}} \text { and }\|\omega\|_{L^{a}}, \text { repectively) }
\end{aligned}
$$




$$
\begin{aligned}
& \leq \frac{1}{4}\|\nabla \omega\|_{L^{2}}^{2}+C_{8}\|\omega\|_{L^{2}}^{2(3-2 \theta-\delta) /(2-2 \theta-\delta)}\|g\|_{L^{r}}^{2 /(2-2 \theta-\delta)} \quad \text { (Young's inequality) } \\
& =\frac{1}{4}\|\nabla \omega\|_{L^{2}}^{2}+C_{8}\|\omega\|_{L^{2}}^{2 /(2-2 \theta-\delta)}\|g\|_{L^{\gamma}}^{2 /(2-2 \theta-\delta)}\|\omega\|_{L^{2}}^{2},
\end{aligned}
$$

where $C_{8}$ depends on $\alpha$ and $\gamma$, and in the above inequality (2.11) we have used the following identities:

$$
\begin{array}{ll}
2 / a+1 / b+1 / \gamma=1, & 1 / b=1 / p-\beta / 3, \\
1 / a=(1-\theta) / 2+\theta(1 / 2-1 / 3), & 1 / p=(1-\delta) / 2+\delta(1 / 2-1 / 3) .
\end{array}
$$

Actually, we can solve (2.12) with

$$
\begin{gathered}
\frac{1}{p}=\frac{\beta}{3}+\frac{1}{2}\left(\frac{1}{2}-\frac{\beta}{3}\right), \quad \frac{1}{a}=\frac{1}{2}-\frac{1}{2} \frac{1}{\gamma}-\frac{1}{4}\left(\frac{1}{2}-\frac{\beta}{3}\right), \\
\frac{1}{b}=\frac{1}{2}\left(\frac{1}{2}-\frac{\beta}{3}\right), \quad \theta=\frac{3}{2}\left(\frac{1}{\gamma}+\frac{1}{2}\left(\frac{1}{2}-\frac{\beta}{3}\right)\right), \quad \delta=\frac{3}{4}-\frac{\beta}{2},
\end{gathered}
$$

where $\alpha$ and $\gamma$ satisfy (1.5). And from (2.12), one has

$$
\beta / 3<1 / b+\beta / 3=1 / p=1 / 2-\delta / 3 \leq 1 / 2,
$$

which implies $\beta<3 / 2$.

Substituting (2.13) into (2.11), we obtain

$$
\left|I_{4}\right| \leq \frac{1}{4}\|\nabla \omega\|_{L^{2}}^{2}+C_{8}\|\omega\|_{L^{2}}^{2 /(\beta+1 / 2-3 / \gamma)}\|g\|_{L^{r}}^{2 /(\beta+1 / 2-3 / \gamma)}\|\omega\|_{L^{2}}^{2},
$$

where $C_{8}$ is a constant depending only on $\alpha$ and $\gamma$.

Putting (2.6), (2.8), (2.9) and (2.14) into (2.5), we have

$$
\begin{aligned}
\frac{d}{d t}\|\omega\|_{L^{2}}^{2}+\|\nabla \omega\|_{L^{2}}^{2} \leq & 4 C_{1}\|\omega\|_{L^{2}}^{2}+2 C_{5}\|\omega\|_{L^{2}}^{4 / 5}\|\omega\|_{L^{2}}^{2} \\
& +2 C_{8}\|\omega\|_{L^{2}}^{2 /(\beta+1 / 2-3 / \gamma)}\|g\|_{L^{\gamma}}^{2 /(\beta+1 / 2-3 / \gamma)}\|\omega\|_{L^{2}}^{2} .
\end{aligned}
$$

So we can use Gronwall's inequality on $\|\omega\|_{L^{2}}$ and it follows from (2.15) that

$$
\begin{aligned}
\|\omega\|_{L^{2}}^{2} & \leq\left\|\omega_{0}\right\|_{L^{2}}^{2} \exp \left\{\int_{0}^{l} 4 C_{1}+2 C_{5}\|\omega\|_{L^{2}}^{4 / 5}+2 C_{8}\|\omega\|_{L^{2}}^{2 /(\beta+1 / 2-3 / \gamma)}\|g\|_{L^{r}}^{2 /(\beta+1 / 2-3 / \gamma)} d s\right\} \\
& \leq\left\|\omega_{0}\right\|_{L^{2}}^{2} \exp \left\{4 C_{1} T+C_{9} T^{3 / 5}+C_{10}\|g\|_{L^{\alpha, \gamma}}^{2 \alpha /(\alpha+2)}\right\},
\end{aligned}
$$

where $C_{1}$ depends on $K, C_{9}$ depends on $K$ and $\left\|u_{0}\right\|_{L^{2}}$, while $C_{10}$ depends on $\alpha, \gamma$ and $\left\|u_{0}\right\|_{L^{2}}$. In (2.16), we have used the energy inequality for $u$, and Hölder's inequality with

$$
\frac{1}{2} \frac{2}{\beta+1 / 2-3 / \gamma}+\frac{1}{\alpha} \frac{2}{\beta+1 / 2-3 / \gamma} \leq 1,
$$


for $2 / \alpha+3 / \gamma \leq \beta-1 / 2$ and $1 / 2 \leq \beta<3 / 2$.

Finally (2.3) follows from (2.15) and (2.16). This completes the proof.

After we have an a priori estimate for $\omega$, the proof of Theorem 1.1 follows from the standard continuation principle, which can be stated as follows.

It is well known [5] that there is a unique strong solution $\tilde{u} \in L^{\infty}\left(0, T_{0} ; H^{1}\left(\mathbb{R}^{3}\right)\right) \cap$ $L^{2}\left(0, T_{0} ; H^{2}\left(\mathbb{R}^{3}\right)\right)$ to $(1.1)$, for some $0<T_{0}$, for any given $u_{0} \in H^{1}\left(\mathbb{R}^{3}\right)$ with $\operatorname{div} u_{0}=0$. Since $u$ is a Leray-Hopf weak solution which satisfies the energy inequality, we have according to the uniqueness result, $u \equiv \tilde{u}$ on $\left[0, T_{0}\right)$. By the a priori estimate (2.3) and the standard continuation argument, the local strong solution $u$ can be extended to time $T$. So we have proved $u$ actually is a strong solution on $[0, T]$. This completes the proof of Theorem 1.1.

\section{Acknowledgements}

The author would like to express sincere gratitude to his supervisor Professor Zhouping Xin for enthusiastic guidance and constant encouragement. Thanks also to the referee for his/her kindly suggestions leading to Remark 1.1. This work is partially supported by Hong Kong RGC Earmarked Grants CUHK-4219-99P and CUHK-4279-00P.

\section{References}

[1] L. Caffarelli, R. Kohn and L. Nirenberg, "Partial regularity of suitable weak solutions of the NavierStokes equations", Comm. Pure Appl. Math. 35 (1982) 771-831.

[2] P. Constantin and C. Fefferman, "Direction of vorticity and the problem of global regularity for the Navier-Stokes equations", Indiana Univ. Math. J. 42 (1993) 775-789.

[3] E. Hopf, "Über die Anfangwertaufgaben für die hydromischen Grundgleichungen", Math. Nachr. 4 (1951) 213-321.

[4] J. Leray, "Étude de divers équations intégrales nonlinearies et de quelques problemes que posent I'hydrodinamique", J. Math. Pures. Appl. 12 (1933) 1-82.

[5] W. von Wahl, The equations of Navier-Stokes and abstract parabolic equations, Aspects of Mathematics E8 (Friedr. Vieweg \& Sons, Braunschweig, 1985).

[6] Y. Zhou, "A new regularity criterion for the Navier-Stokes equations in terms of the gradient of one velocity component", Methods Appl. Anal. 9 (2002) 563-578.

[7] Y. Zhou, "A new regularity criterion of weak solutions to the Navier-Stokes equations", preprint, 2002.

[8] Y. Zhou, "Regularity criteria in terms of pressure for the 3-D Navier-Stokes equations in a generic domain", Math. Ann. 328 (2004) 173-192. 